\title{
Presencia de las redes sociales en los museos argentinos de gestión nacional

\author{
Avances significativos en su implementación \\ (2015-2017)
}

\section{( Marcela Beatriz Ristol \\ Universidad Nacional de Mar del Plata. Facultad de Humanidades. Mar del Plata, Argentina | marcelaristol@gmail.com / mristol@mdp.edu.ar / ORCID https://orcid.org/oooo-0003-3765-6604}

\section{Resumen}

Los recursos Web 2.0 favorecen la comunicación y posibilitan, entre otros aspectos, la interacción con la comunidad. La presencia de las redes sociales en las instituciones museísticas es todavía un desafío para la acción cultural. En una investigación previa, año 2015, se analizó la visibilidad de las redes sociales en los museos argentinos. Los principales resultados proporcionaron una escasa aplicación de este recurso. En esta oportunidad se examina, con relación al 2017, la existencia de avances significativos en aspectos que reconcilien su implementación.

Presence of social networks in Argentine Museums of National Management. Significant progress in its implementation (2015-2017)

\begin{abstract}
The Web resources 2.0 favor the communication and make possible, between other aspects, the interaction with the community. The presence of the social networks in the museums is still a challenge for the cultural action. In a previous investigation, of 2015, analyzed the visibility of the social networks in the Argentine museums was analyzed. The main results provided a scarce application of this resource. In this opportunity, in relation to 2017 , if significant advances exist in aspects that reconcile its implementation are examined.
\end{abstract} Palabras clave Redes Sociales Visibilidad Museos Argentina

Keywords

Social Networks

Visibility

Argentine

Museums 
1. Bernardino de la Trinidad González Rivadavia y Rivadavia 1780-1845. Primer Presidente de las Provincias Unidas del Río de la Plata siendo su mandato 1826-1827.

\section{Museos Argentinos. Los inicios}

El inicio de los museos argentinos se remonta a la creación del Museo Argentino de Ciencias Naturales (MACN), sito en la ciudad de Buenos Aires, dispuesta por la Asamblea General del año 1812. De esta manera se estableció, en paralelo al primer museo público, la naciente y más antigua institución científica de la República Argentina. Esta iniciativa, ideológica y política, estuvo liderada por Bernardino Rivadavia ${ }^{1}$, quien afianzó los términos del coleccionismo y gestionó ese acervo para crear el fondo del museo, con el apoyo de las donaciones que realizó Bartolomé Doroteo Muñoz (Ministerio de Ciencia, Tecnología e Innovación Productiva [MINCYT], 2017).

Si bien este fue el primer antecedente, los próximos años, cercanos a la segunda mitad del siglo XIX se consolidaron en una impronta más sistemática. Como marco ideológico de estas experiencias, entre los años 1850 y 1880, la Argentina manifestaba un gradual abandono de cánones hispano-criollos, los que se procuraba "reemplazar con nuevos valores laicos y republicanos" (Risnicoff de Gorgas, 2006: 3).

Una representación más amplia del contexto que facilitó el surgimiento de estas instituciones en Argentina se aprecia en el origen del Museo Histórico Nacional:

Luego de medio siglo de guerras civiles, la Argentina se embarcó en el proceso de consolidación de su Estado-Nación. La rápida expansión económica, el desarrollo de las comunicaciones y la llegada masiva de inmigrantes cambiaron por completo la composición socio-económica del país y Buenos Aires, en particular, se transformó en una ciudad moderna y cosmopolita. Fue entonces cuando los grupos dirigentes se enfrentaron con la necesidad de desarrollar un sentimiento de nacionalidad que, ante los acelerados y profundos cambios que estaban ocurriendo, lograra unificar a la sociedad argentina apelando a un acervo común de tradiciones y a una historia compartida (Museo Histórico Nacional [MHN], 2020).

Los nacientes museos argentinos afianzaron la imagen del Estado-Nación. En esa época se construyó la idea de legitimar las instituciones, en semejanza con otras, a fin de fortalecer los principios del "ciudadano, y de identidad nacional" (Risnicoff de Gorgas 2006:3), ${ }^{2}$ así lo describe este autor cuando indica que se refleja una conciencia nacional homogénea en una población con diversas raíces. Y a su vez, la necesidad de crear nuevos hábitos civiles (Podgorny, 2005), dictados por la Nación y dirigidos especialmente a los escolares e hijos de los inmigrantes, orientados a transmitir un único criterio en la transferencia de valores y sentimientos patrióticos. Estas premisas, hechos fundantes de la historia argentina, impulsaron la creación del Museo Histórico Nacional (Malosetti Costa, 2010: 75).

Américo Castilla (2010), en tanto, expone que estas instituciones fueron necesarias para "consolidar la personalidad de las nuevas naciones"y así establecer núcleos de instituciones con vista independentista.

Uno de los legados patrimoniales más significativos del territorio del Río de la Plata ${ }^{3}$ fue el aporte que realizó la Orden Jesuítica. Los jesuitas se establecieron en el territorio a fines del siglo XVII y organizaron un sistema complejo desde lo cultural y económico con importancia histórica regional. "Ocupaban grandes extensiones en lo que hoy es Argentina, Uruguay, Brasil y Paraguay, con ramificaciones en Chile y Bolivia." (Risnicoff de Gorgas, 2006: 4). En la actualidad, su acervo más destacado forma parte de las colecciones del Museo Histórico Nacional, de la Biblioteca Mayor y del Museo Histórico de la Universidad Nacional de Córdoba. 
Una de las peculiaridades que se generaron en los museos de América fue su vinculación con universidades, como es el caso del Museo de Ciencias Naturales con la Universidad Nacional de La Plata. El Museo había sido creado en el año $1884 \mathrm{su}$ fundador y primer director fue Francisco Pascasio Moreno4. El Museo de La Plata "surgió de la donación de un particular vivo y en pleno uso de sus facultades, con la singularidad de haberse incluido, dicho donante, como parte de su voluntad: el estado aceptó esas colecciones y los objetos pero también el control de las mismas por la mirada vigilante del donador" (Podgorny, 2005: 238).

A principios del siglo XX, en el año 1906, el "Museo, dirigido entonces por Samuel Lafone Quevedo (1835-1920), pasó a depender administrativamente de la Universidad Nacional de La Plata, agregando a las funciones originales de exhibición pública e investigación, la de formación académica, bajo la denominación general de Instituto del Museo-Facultad de Ciencias Naturales" (Universidad Nacional de La Plata [UNLP], 2020).

Esta relación asociada a la materialidad científica y utilizada a manera de instrumento didáctico para la enseñanza, le valió para ser reconocido más como gabinete de curiosidades que como museo. Esta cualidad es mencionada por algunos autores que han estudiado el período Fernández Balboa (2009), Achim y Podgorny (2014), Lopes (2010), Malosetti Costa (2010), Castilla (2010) entre otros y definen el comienzo de los museos argentinos como "gabinetes de curiosidades" integrados en un primer momento por elementos coleccionados previamente por personas entendidas y donados a ellos.

Otros tipos de museos, de arte e historia, surgieron a fines del siglo XIX: el Museo Histórico Nacional (MHN) en 1889 y el Museo Nacional de Bellas Artes (MNBA), ${ }^{6}$ en 1895 . La impronta de estas instituciones se vincula con ideas nacionalistas de la época, enraizadas en los hombres de mayo (Malosetti Costa, 2010), que pensaban una patria independiente de España. Sus colecciones originales fueron objetos donados por personalidades destacadas de la época, los héroes de Mayo. ${ }^{7}$ Sus primeros directores fueron A. P. Carranza ${ }^{8}$, en el caso del MHN, y Eduardo Schiaffino, ${ }^{9}$ en el MNBA. Este último promovió instalar el arte nacional en concordancia con los museos europeos de la época.

Así lo entendía también el coleccionista Juan B. Sosa ${ }^{10}$, quien donó en vida 50 obras de arte para que sirvieran de base a una pinacoteca pública. Un segundo mecenas, Adriano Rossi ${ }^{11}$, hizo lo propio con 81 cuadros de su colección privada de arte. Estos legados posibilitaron la apertura del museo, conjuntamente con el patrocinio del centro "El Ateneo" (Laumonier, 1993).

Los museos pedagógicos decimonónicos, en tanto, surgieron para acompañar la formación profesional de maestros y en muchos casos la construcción de los sistemas educativos nacionales. En el caso argentino, los primeros fueron pensados también a fines del siglo XIX; en 1883 se creó el Museo Escolar Nacional y que fue establecido en 1889 .

El museo contaba con objetos, libros de didáctica y mapas, instrumentos de madera para la enseñanza de la geometría. A partir de las exposiciones del Centenario (1910), por iniciativa del Doctor Ramos Mejía, se organizó el Museo Escolar Sarmiento que funcionó hasta la década de 1940 en la Escuela Normal Nº 9 "Domingo Faustino Sarmiento". En 1929 la maestra y directora Rosario Vera Peñaloza creó y dirigió en el Instituto Félix Fernando Bernasconi, los Museos "Geográfico: Dr. Juan B. Terán” y de "Ciencias Naturales: Dr. Ángel Gallardo" específicamente dirigidos a los alumnos de las escuelas primarias (Museo de las Escuelas, 2020).
4. Francisco Pascasio Moreno (1852-1919). Científico naturalista argentino, explorador de la Patagonia. Fue además escritor, diputado nacional y vicepresidente del Consejo Nacional de Educación.

5. Museo Histórico Nacional https://museohistoriconacional.cultura.gob.ar/ [Consulta: 1 marzo 2020]

6. Museo Nacional de Bellas Artes: http://www.mnba.gob. ar/ [Consulta 1 marzo 2020]

7. Se refiere a los patriotas que intervinieron en la Revolución de Mayo de 1810

8. "Adolfo Pedro Carranza nació el 7 de agosto de 1857 , fue un historiador y abogado argentino, creador del Museo Histórico Nacional de su país y director del mismo durante 25 años. ... Murió el 14 de agosto de 1914, en Buenos Aires." (Todo Ciencia.com.ar, 2020).

9. Eduardo Schiaffino (1858-1935). Primer director del Museo Nacional de Bellas Artes, Argentina.

10. Juan Benito Sosa (1839-1909). "El tipo de coleccionista encarnado por Sosa fue excepcional ya que, más que por deleite personal, adquirió sus obras con el propósito explícito de ofrecerlas al gobierno. La distinción social no descansó en primera instancia en la posesión y exhibición privada de las pinturas sino - desde su génesis - en el hecho de hacerlas públicas." (Baldasarre, 2006: 301).

11. "Este fue el caso del descendiente de italianos Adriano Rossi (1814-1893), quien efectuó un año antes de su muerte la donación fundadora de lo que sería posteriormente el MNBA". (Baldasarre, 2006: 298)

12. “En 1893, junto con Severo Rodríguez Etchart, Eduardo Sívori, Ernesto de la Cárcova, Augusto Ballerini, Graciano Mendilaharzu, Ángel Della Valle, entre otros, creó el Ateneo, entidad organizadora de las primeras exposiciones de arte argentino y desde donde se impulsó la creación del Museo Nacional de Bellas Artes." (Museo Nacional de Bellas Artes [MNBA], 2020). 
Transcurrido más de un siglo desde la creación del primer museo público argentino, el panorama actual es totalmente diferente en cuanto al incremento del patrimonio cultural de nuestro país. Así lo manifiesta el antropólogo argentino, Marcelo Weissel:

El mapa cultural de la Argentina presenta 27 Museos Nacionales, 17 patrimonios de la humanidad, 950 monumentos y lugares históricos de 6.000 bienes declarados patrimonio nacional, 900 espacios de exhibición patrimonial..." A su vez, "se desarrollan cuatro grandes rutas culturales que conectan 35 sitios en la ruta de las Ruinas de Estancias Jesuitas, 24 sitios en los Valles Calchaquíes, 17 sitios siguiendo la ruta de la Yerba Mate, y 31 sitios con la Ruta de la Independencia. Este patrimonio es eje de promoción estratégica con los países del Mercosur, o bien con los países andinos con quienes se presentó la nominación del Camino Principal Andino o Qhapaq Ñan integrando a 15.000 kilómetros de inventarios en 6 países" (Weissel, 2014: 30).

\section{Redes Sociales}

En la actualidad Internet es el principal instrumento tecnológico en la vida de las personas y, cada vez más, interviene en los cambios que se producen en la comunicación.

Como indica Jesús Martín-Barbero (2002) la tecnicidad mediática como dimensión estratégica de la cultura, configura un nuevo sistema comunicativo; un ámbito diferente de socialización, que permite la circulación de información, promueve el intercambio de experiencias, habilita proyectos que hacen posible un nuevo entorno comunicacional, entre sus principales influencias.

En relación con las redes sociales existen factores indiscutibles sobre los beneficios aportados por los recursos de la web 2.0, tales como la horizontalidad discursiva en la comunicación que facilita nuevas posibilidades en los procesos de interacción, participación, construcción de mensajes y, además, proporciona un contacto inmediato y directo sin intervención de mediadores.

El modelo de la comunicación intervenida por redes digitales, transforma las relaciones entre emisores y receptores, y su posibilidad de alcance inimaginable es una de las características revolucionarias de estos recursos. En palabras de Manuel Castells (2014)

"La clave del éxito no es el anonimato, sino más bien la autopresentación de una persona real que está conectada con personas reales....Por tanto, estamos ante una sociedad autoconstruida mediante la conexión en red con otras redes. Pero no se trata de una sociedad virtual. Existe una estrecha conexión entre las redes virtuales y las redes vivas. Es un mundo híbrido, un mundo real. No es un mundo virtual ni un mundo aparte." (p. 140) Es decir, "todos viven su vida física, pero se conectan cada vez más y en múltiples dimensiones a las redes sociales" ( $p .141$ ).

Estudiar sus rasgos más significativos, aplicados a los museos, implica entre sus tantas variables, conocer su trascendencia en este contexto, teniendo en cuenta que, en su origen, no fueron creadas para este fin y por ende se desconoce su eficacia y todas las posibilidades de uso que ofrecen para este tipo de organizaciones. Es decir, las redes sociales al servicio de la comunicación de los museos. 


\section{Metodología}

Este trabajo se basa en un estudio del año $2015^{13}$, en el que se analizó el total de las instituciones informada por la Guía Nacional de Museos (MUS) (2013), en relación a la visibilidad de las redes sociales en los museos argentinos (Ristol, 2015).

En esta ocasión, se consideró particularizar el análisis en los museos de gestión nacional por ser aquellos que se encuentran más provistos de recursos y de personal, y por su vinculación con el Ministerio de Cultura de la Presidencia de la Nación, de modo que merecen una especial atención. Si bien actualmente se informan modificaciones en estas instituciones, para este estudio se determinó realizar la indagación comparativa con los museos observados en el 2015.

En consecuencia, se examina la situación de 25 museos descriptos en la investigación anterior, identificados como de gestión nacional. El propósito de este trabajo es medir los cambios producidos en la visibilidad de los museos argentinos de dicha gestión, a partir de los elementos que proporcionan las propias redes sociales. La observación de las plataformas se efectúa desde la perspectiva del usuario, de modo que, la información obtenida está restringida a los datos que proporcionen los museos en la web.

El análisis se formaliza en dos de las redes con mayor repercusión, según las líneas de los museos argentinos: Facebook y Twitter.

La Tabla 1 presenta la totalidad de los museos de gestión nacional correspondiente al estudio llevado a cabo en el 2015 y, en paralelo, se enumera la totalidad de los museos comunicados por el Ministerio de Cultura de la Nación en 2017 (Ministerio de Cultura, 2017), con el propósito de mostrar los actuales.

Por otra parte, es pertinente aclarar que en el periodo de indagación la página oficial del Ministerio de Cultura hace públicos los museos identificados en la Tabla 1, y además añade una clasificación distando museos e instituciones (Ministerio de Cultura, 2017), condición no mencionada anteriormente. Asimismo, se reconocen otros que no figuraban en la Guía MUS, como: Museo del Libro y de la Lengua, Museo Malvinas e Islas del Atlántico Sur, Casa Nacional del Bicentenario, Casa Creativa del Sur y Casa Central de la Cultura Popular. En cuanto al Museo del Bicentenario, continúa, pero cambia su denominación a Museo Casa Rosada.

En relación a la asignación de variables cuantitativas más apropiadas para la exploración comparativa se efectúa una selección de los elementos definidos en el estudio anterior, con el objetivo de acercar más relevancia a los resultados. Estas estructuras se agrupan en niveles superiores representadas por cuatro categorías: Informativa, Gestión, Usufructo de la herramienta y Fidelización/Interacción.

En consecuencia se decidió descartar varias que carecen de interés, por ejemplo inicio de la cuenta del museo que no conforma relevancia para esta investigación. ${ }^{14}$

La Tabla 2, muestra la elección de los elementos constitutivos para las dos redes Facebook y Twitter, allí se aprecian las variables asignadas para la investigación actual.
13. La muestra fue de 1.005 museos. 
Tabla 1- Museos argentinos de gestión nacional (2015-2017)

\begin{tabular}{|c|c|}
\hline Guía Museo, 2013 (Investigación 2015) & Ministerio de Cultura, 2017 \\
\hline Complejo Histórico Cultural Manzana de las Luces & Complejo Histórico Cultural Manzana de las Luces \\
\hline Estancia de Jesús María - Museo Jesuítico Nacional & Estancia de Jesús María - Museo Jesuítico Nacional \\
\hline Museo Casa de Ricardo Rojas & Museo Casa de Ricardo Rojas \\
\hline Museo “Casa de Yrurtia” & Museo "Casa de Yrurtia" \\
\hline Museo Casa Histórica de la Independencia & Museo Casa Histórica de la Independencia \\
\hline $\begin{array}{l}\text { Museo de la Estancia Jesuítica de Alta Gracia y Casa } \\
\text { del Virrey Liniers }\end{array}$ & $\begin{array}{l}\text { Museo de la Estancia Jesuítica de Alta Gracia y Casa } \\
\text { del Virrey Liniers }\end{array}$ \\
\hline Museo del Hombre & Museo del Hombre \\
\hline Museo Evita & Museo Evita \\
\hline Museo Histórico del Norte & Museo Histórico del Norte \\
\hline Museo Histórico Nacional & Museo Histórico Nacional \\
\hline $\begin{array}{l}\text { Museo Histórico Nacional del Cabildo y de la } \\
\text { Revolución de Mayo }\end{array}$ & $\begin{array}{l}\text { Museo Histórico Nacional del Cabildo y de la } \\
\text { Revolución de Mayo }\end{array}$ \\
\hline Museo Histórico Sarmiento & Museo Histórico Sarmiento \\
\hline Museo Mitre & Museo Mitre \\
\hline Museo Nacional de Arte Decorativo & Museo Nacional de Arte Decorativo \\
\hline Museo Nacional de Arte Oriental & Museo Nacional de Arte Oriental \\
\hline Museo Nacional de la Historia del Traje & Museo Nacional de la Historia del Traje \\
\hline Museo Nacional del Grabado & Museo Nacional del Grabado \\
\hline Museo Regional de Pintura “José A. Terry” & Museo Regional de Pintura “José A. Terry” \\
\hline Museo Roca - Instituto de Investigaciones Históricas & Museo Roca - Instituto de Investigaciones Históricas \\
\hline Museo y Biblioteca Casa Natal de Sarmiento & Museo y Biblioteca Casa Natal de Sarmiento \\
\hline $\begin{array}{l}\text { Museo y Biblioteca de la Casa del Acuerdo de San } \\
\text { Nicolás }\end{array}$ & $\begin{array}{l}\text { Museo y Biblioteca de la Casa del Acuerdo de San } \\
\text { Nicolás }\end{array}$ \\
\hline Palacio Nacional de las Artes (ex Palais de Glace) & Palacio Nacional de las Artes (ex Palais de Glace) \\
\hline $\begin{array}{l}\text { Palacio San José - Museo y Monumento Nacional Justo } \\
\text { José de Urquiza }\end{array}$ & $\begin{array}{l}\text { Palacio San José - Museo y Monumento Nacional } \\
\text { Justo José de Urquiza }\end{array}$ \\
\hline Museo Nacional de Bellas Artes - MNBA & Museo Nacional de Bellas Artes - MNBA \\
\hline Museo del Bicentenario & $\begin{array}{l}\text { No se informa en el sitio. En la web se denomina } \\
\text { Museo Casa Rosada }\end{array}$ \\
\hline--- & Museo Malvinas e Islas del Atlántico Sur \\
\hline--- & Casa Nacional del Bicentenario \\
\hline-- & Museo del Libro y de la Lengua \\
\hline--- & Casa Creativa del Sur \\
\hline--- & Casa Central de la Cultura Popular \\
\hline
\end{tabular}


Tabla 2 - Variables asignadas a la investigación. 2017

\begin{tabular}{ll}
\hline Red Social Facebook & Variables \\
\hline & Utiliza tendencia \\
& Cantidad de seguidores \\
& Opinión de los seguidores \\
& Sube videos \\
& Genera eventos \\
& Visitas registradas \\
\hline Red Social Twitter & Variables \\
\hline & Frecuencia de utilización \\
& ¿Es una cuenta oficial o verificada? \\
& Saca foto o videos \\
& Cantidad de fotos y videos \\
& Seguidores \\
& Siguiendo \\
Cantidad aproximada de Tweets por día
\end{tabular}

Fuente: elaboración propia

\section{Resultados}

\section{Sobre Facebook}

Del total de 25 museos $^{15}, 21$ registran cuenta en Facebook. Al 2017 se comprueba que el Museo Casa de Yrurtia no dispone de contenido en la red y, por otro lado, se evidencia la activación de nuevas cuentas: Museo Nacional del Grabado y Museo Nacional del Hombre. En cuanto al Museo Regional de Pintura "José A. Terry" continúa en la red con perfil amigo. ${ }^{16}$

Hay que considerar que en los últimos años la red promueve modificaciones que reorganizan diferentes secciones con la finalidad de brindar más dinámica al recurso. Entre ellas Publicaciones y comunidad, esta última administra información concerniente al total de me gusta / de seguidores / de visitas.

El primer elemento observado Utiliza tendencia $(\#)^{17}$ creció un $13 \%$ respecto al periodo anterior, no obstante es válido aclarar que solamente lo emplea el $33 \%$ de los museos.

El Gráfico 1, muestra la cantidad de Seguidores ${ }^{18}$ que en el período observado presenta un crecimiento del $34 \%$. Además, hay que mencionar que el único museo con diferencia negativa, en representación de Seguidores, es el Museo Roca-Instituto de Investigaciones Históricas, en referencia al estudio del 2015 contaba con 1.127 y en el presente informa 118. En cuanto a la Opinión ${ }^{19}$ se mantiene el mismo porcentaje.

15. Museos de gestión nacional registrados en el año 2015 .

16. Para este tipo de instituciones se recomienda la activación de la red en página y no en perfil amigo.

17. Utiliza tendencia: se muestra en el muro de la red y se define como temas y hashtags (\#) es lo suficientemente relevante para que el usuario pueda filtrar y encontrar conversaciones similares.

18. Número de seguidores: cantidad de usuarios vinculados intencionalmente con la página.

19. Opinión de los seguidores: ponderación de los usuarios, medida en estrellas (se califica de o a 5). El dato se encuentra en "opiniones" y se clasifica en: Medida de opinión: del o al 5 . No hay opinión: los usuarios no calificaron la red. No tiene: no figura en la red el término opinión. 
Gráfico 1. Red Facebook cantidad de seguidores, periodo 2015-2017

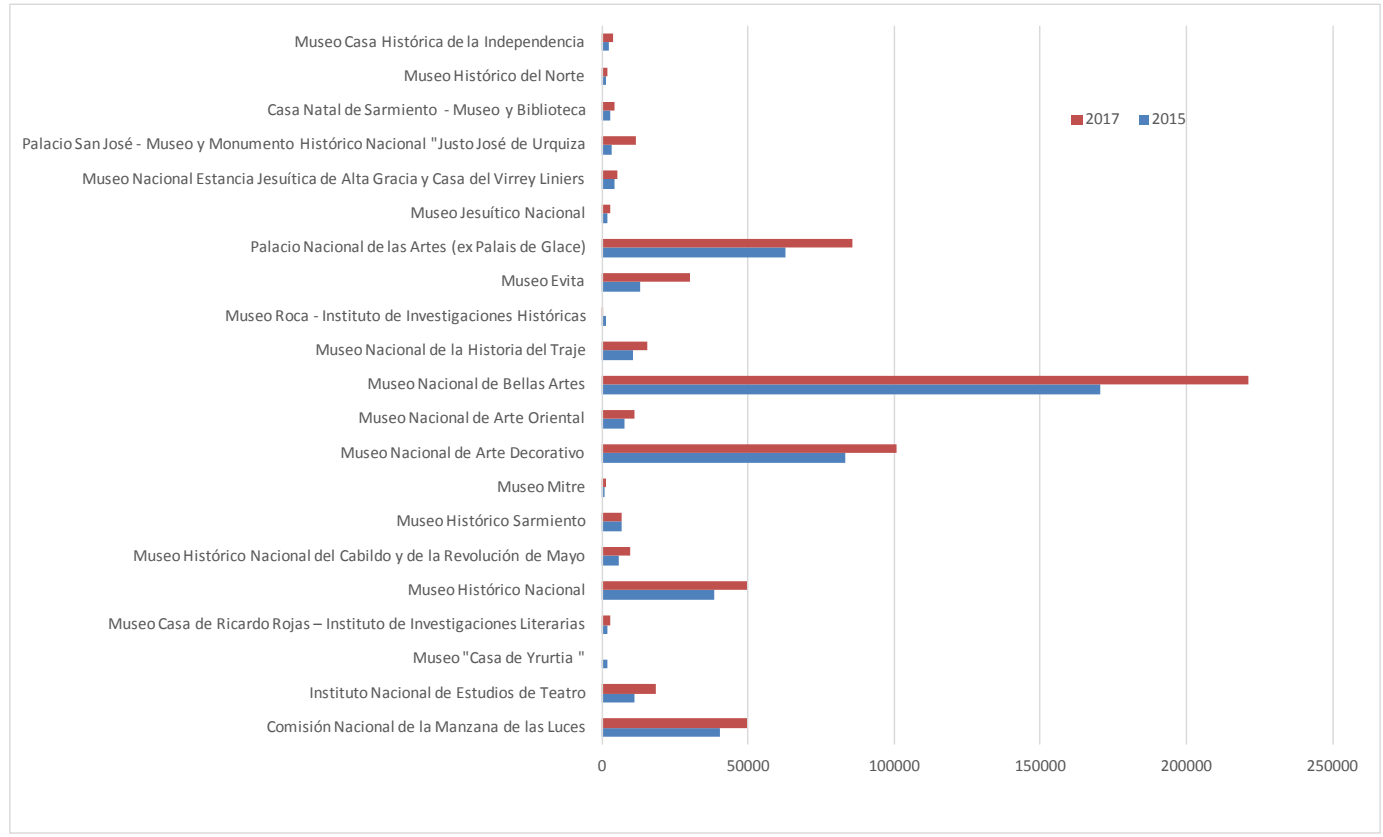

Fuente: Elaboración propia

20. Sube videos: publica video/s en la red

21. Como se indicó anteriormente, cerró su cuenta en la red Facebook.

22. Genera eventos: creación de sucesos programados.

23. Personas que registraron visitas aquí: cuenta las visitas de usuarios que etiquetaron el lugar.

24. Frecuencia de utilización: número de veces que se emite un tweet. Se confirmó el dato de forma manual y de acuerdo con la siguiente escala: Diaria, todos los días. Semanal, se emite de 1 a 3 veces por semana. Mensual, produce y publica con una frecuencia de una vez por mes y se cuenta en esta escala, hasta una vez cada tres meses. Esporádica, la frecuencia es una por año. Nula, más de un año de inactividad.
En relación al elemento Sube videos ${ }^{20}$, se observa que el $86 \%$ hace uso de este recurso, es decir solo 3 museos no aplican, considerando que entre ellos se encuentra el Museo Casa de Yrurtia ${ }^{21}$. A su vez, es significativo el incremento en el periodo estudiado, del $34 \%$. Las razones de estos cambios se desconocen, no obstante, se puede aludir a la importancia que actualmente tiene la realización de videos en directo promocionado en diversas plataformas. En la misma coyuntura se encuentra el dispositivo Genera eventos ${ }^{22}$, con un porcentaje de 86, es decir, 18 museos emplean el recurso. En estos años se sumaron 3.

El Gráfico 2 cuantifica el número de Visitas registradas ${ }^{23}$ por los usuarios de la red. Se aprecia una diferencia bien marcada entre aquellos con registros que superan las 10.000 visitas y un conjunto mayoritario de museos concentrados en un rango más pequeño de o a 6.000.

\section{Sobre Twitter}

Continuando con el criterio establecido para la red anterior, en el año 2015 se observaron 13 instituciones con red Twitter. A diferencia de Facebook, en 2017 no se encontraron bajas, por el contrario, se observan altas: Museo y Biblioteca Casa Natal de Sarmiento y Museo y Biblioteca de la Casa del Acuerdo de San Nicolás. Por lo que se advierte un incremento en la apertura de cuentas del $8 \%$.

Acerca de la variable Frecuencia de utilización ${ }^{24}$ se comprueba un cambio significativo de esporádica a mensual en el Museo Nacional Estancia Jesuítica de Alta Gracia y Casa del Virrey Liniers, y de esporádica a diaria en el Museo Roca-Instituto de Investigaciones Históricas. En cuanto a las demás instituciones, 77 \%, permanecen en la Frecuencia de utilización diaria. 
Gráfico 2 Red Facebook visitas registradas, periodo 2015-2017

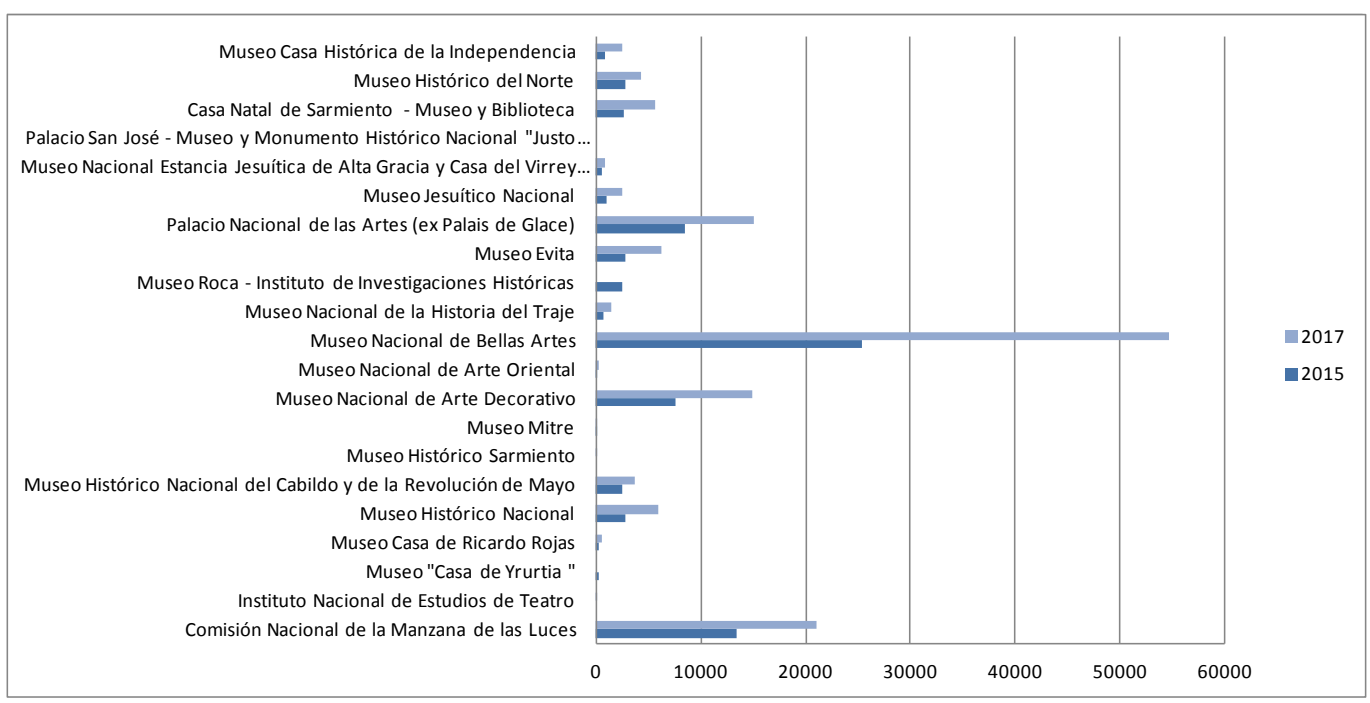

Fuente: Elaboración propia

En cuanto a la Verificación de la cuenta ${ }^{25}$ continúa 1 de los 13 museos: el Nacional de Bellas Artes.

En relación al elemento Saca fotos o videos ${ }^{26}$, el único que no manifestaba actividad en la indagación anterior correspondía al Museo Roca-Instituto de Investigaciones Históricas. En el 2017 se corrobora la información y presenta movimiento con 83 videos publicados. El resto de los museos, registra un crecimiento global del $87,11 \%$. Además, acerca de la variable Cantidad de fotos y videos ${ }^{27}$, el caso más revelador se le asigna al Museo Histórico Nacional del Cabildo y de la Revolución de Mayo que incrementó más de siete veces el volumen de elementos publicados, de 50 a 450 .

Los elementos Seguidores y Siguiendo ${ }^{28}$ conviven en la red promoviendo diferentes relaciones entre sus miembros. La simetría o reciprocidad permiten alianzas equilibradas. El mayor aumento se produjo en el Museo Roca, de 45 a 299 y el Museo Mitre cuadruplicó sus seguidores, de 206 a 1.174. El primer caso, si bien quintuplicó la relación, el número final no representa relevancia en cuanto a conformar visibilidad en los términos de una institución de gestión nacional y de alto nivel cultural.

El Gráfico 3 muestra la Cantidad de seguidores que poseen los museos. Se observa que el grupo mayoritario continúa con menos de 5.000 y solamente dos instituciones superan los 10.000, a saber, Museo Nacional de Bellas Artes, con 38.200 y el Palacio Nacional de las Artes, con 16.600. Todos ellos de arte.

A la vez, se observa que la única institución sin actividad desde el año 2016 es el Museo del Bicentenario, si bien se desconoce las causas de la inactividad se
25. ¿Es una cuenta oficial o verificada? se usa para establecer la autenticidad de la cuenta. Puede decir "cuenta oficial" o "cuenta verificada", en este último caso, se indica mediante una insignia de color azul.

26. Saca fotos o videos: publica fotografía/s en la red.

27. Cantidad de fotos o videos: número de fotografías y videos publicados

28. Seguidores, también llamados Followers. Recibe tweets en su página de inicio y a diferencia de otras redes sociales, la solicitud de un follower no es recíproca. Siguiendo, también se denomina Following. Seguir a alguien en Twitter. 
Gráfico 3. Red Twitter cantidad de seguidores, periodo 2015-2017

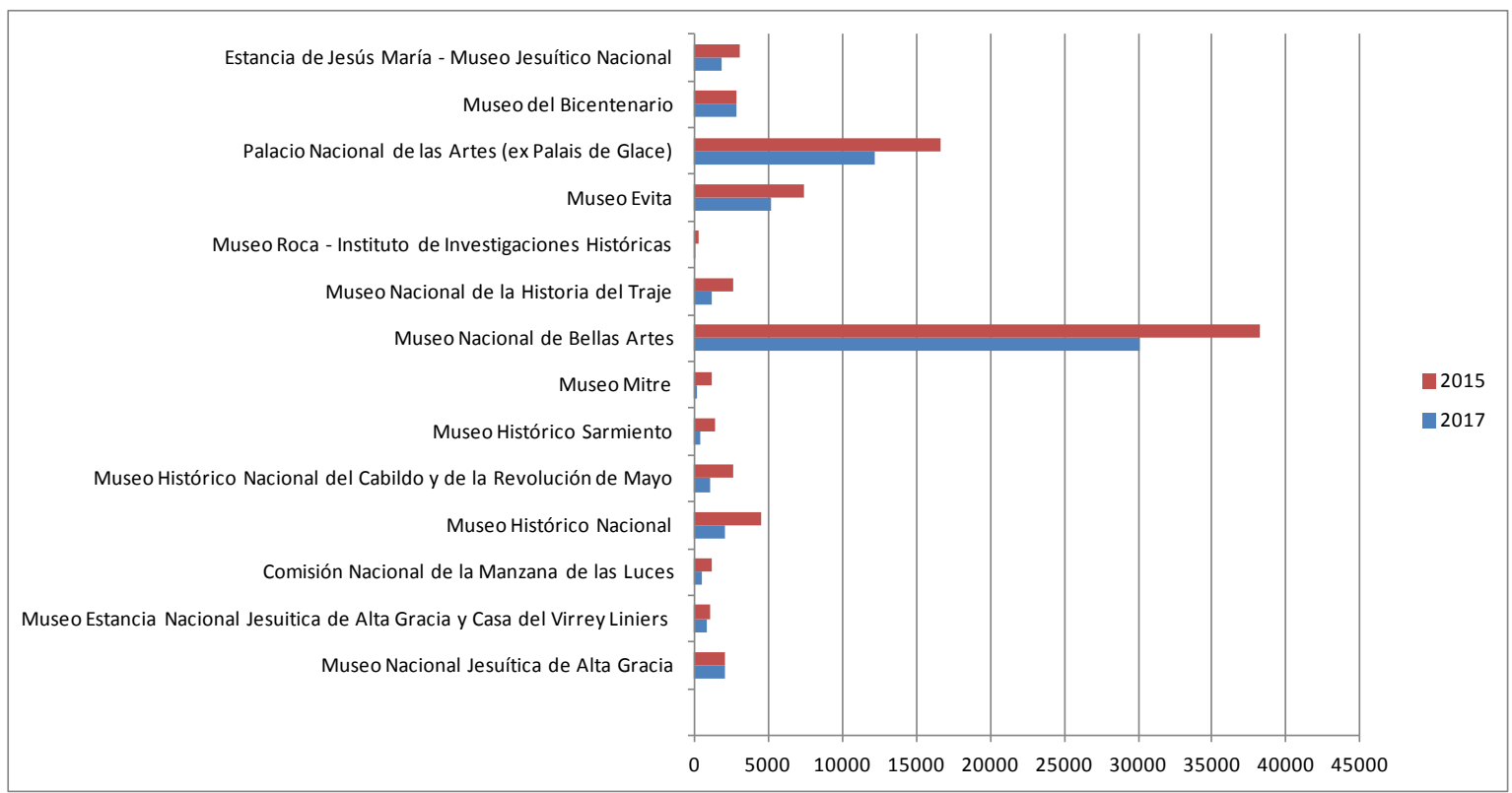

Fuente: Elaboración propia

29. Cantidad aproximada de Tweets por día: se evalúa la emisión de tweets, solo en el caso que la frecuencia de utilización sea diaria. conjetura que uno de los factores puede ser el cambio de nombre, como se indicó anteriormente, hoy se denomina Museo Casa Rosada.

Por último, en el Gráfico 4, se registra Cantidad aproximada de Tweets por día ${ }^{29}$, allí se observa la misma asiduidad que en el período anterior.

\section{Conclusiones}

En líneas generales, existe un crecimiento en la variación porcentual de los elementos. En la red Facebook los casos más representativos se manifiestan en Sube video y Genera eventos, mostrando la conveniencia que produce este tipo de servicios. Es tendencia en las plataformas de recursos 2.0 la utilización de streaming, como la promovida por Facebook Live. Por consiguiente, se recomienda que los museos se adecúen y aprovechen las herramientas que ofrecen estos medios, tal como incrementar el uso de hashtags para conformar términos que, asociados a la red de los museos, generen posibles preferencias. A su vez, se comprueba el cierre de la red del Museo Casa de Yrurtia, por lo menos con la misma identificación, dejando un espacio de interacción con la comunidad y, en contraposición, se observa la activación de dos nuevas cuentas con buenas perspectivas.

Respecto a la red Twitter se aprecia la apertura de nuevos medios, en virtud de lo cual se infiere que los museos eligen consolidarse en estos canales. No obstante, continúan sin declarar las cuentas como oficiales, el único con mención verificada es el Museo Nacional de Bellas Artes. La insignia azul de verificación sirve para confirmar la autenticidad y desde un punto de vista prestigia a la cuenta, aunque es válido aclarar que este proceso no es fácil de gestionar. Acerca del elemento Frecuencia de utilización, 
Gráfico 4. Cantidad aproximada de Tweets por día, 2015-2017

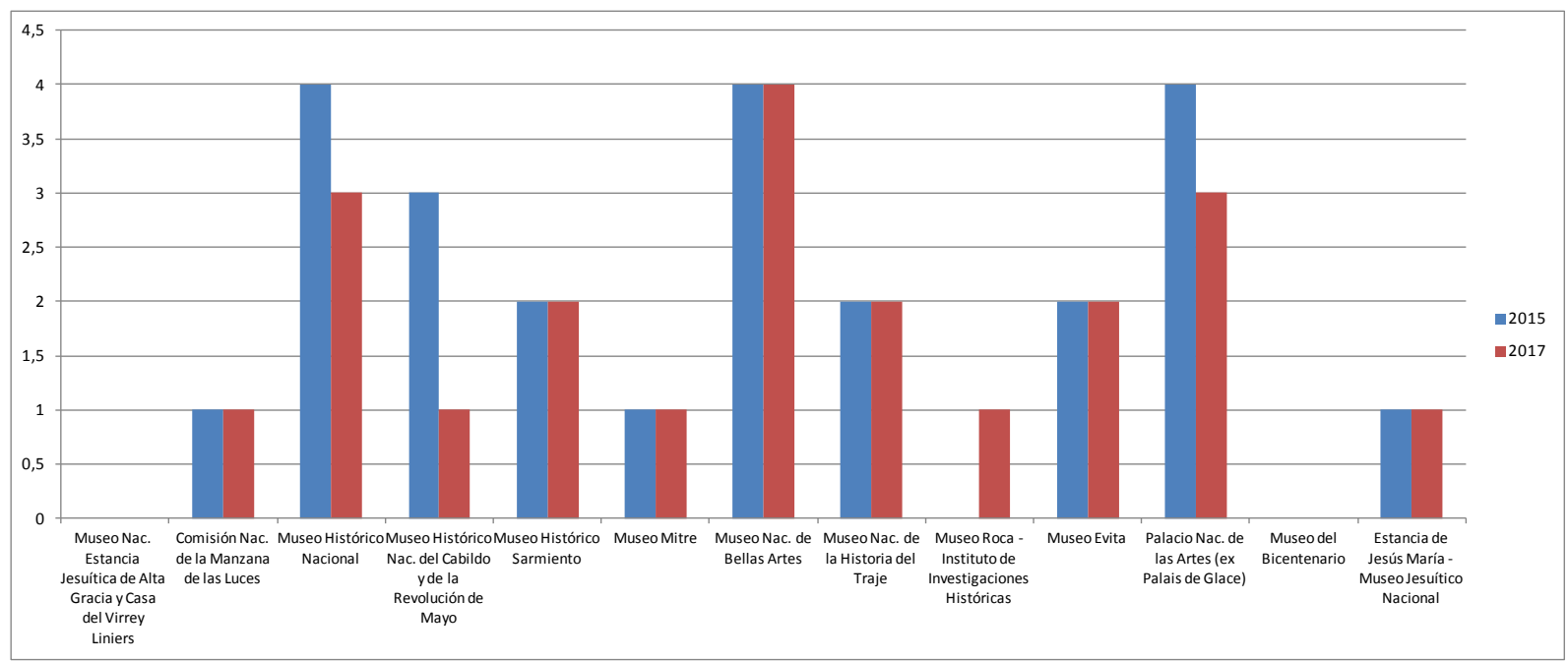

Fuente: Elaboración propia

en su mayoría permanecen en la asiduidad diaria. Esto refleja un mecanismo continuo de tweets en el medio, lo cual proporciona visibilidad al museo, más, teniendo en cuenta que la construcción de los mensajes involucra tiempo y dedicación de recursos, factores que las instituciones no siempre poseen. Otro rasgo que evidencia un alto grado de utilización es el de Saca fotos y videos que registra un crecimiento global del $87,11 \%$. Asociado a este concepto corresponde el de Cantidad de fotos y videos, siendo el caso más revelador el Museo Histórico Nacional del Cabildo y de la Revolución de Mayo con un incremento de siete veces el volumen de elementos publicados.

En relación con el número de Seguidores en las redes analizadas se observa que, para los administradores de los museos, continúa siendo un desafío incursionar en acciones que faciliten un aumento de adeptos. La gran mayoría de las instituciones no alcanza los 10.000 seguidores. En este sentido, ampliar la comunidad de la red implica gestionar acciones continuas que interactúen con el público para que se hable de la entidad e influir en el entorno. Para eso, la misma red proporciona patrones con diferentes sugerencias para incrementar el volumen de usuarios, este sería un punto a tener en cuenta. No obstante, se infiere que la activación y posterior seguimiento de cierto perfil en la red, supone también asignar recursos de todo tipo y de manera permanente.

En conclusión, se percibe un crecimiento en los valores de los elementos analizados, aunque debe señalarse que para optimizar el rendimiento global, se debería enriquecer la propuesta, valiéndose de las propias herramientas que cada red ofrece. Los museos de gestión nacional, son de los mejor dotados ediliciamente, en cuanto a su personal, sus recursos, su gestión y más desarrollados en el universo $w e b$, correspondería generar presencia posibilitando una mejor visibilidad en las plataformas 2.0. 


\section{Q Referencias bibliográficas}

》 Achim, Miruna e Irina Podgorny, eds. 2014. Museos al detalle. Colecciones, antigüedades e historia natural, 1790-1870. Rosario: Prohistoria Ediciones.

»Baldasarre, María Isabel. 2006. Sobre los inicios del coleccionismo y los museos de arte en la Argentina. En Anais do Museu Paulista. História e cultura material. Vol.14, no. 1, 293-321. <https://doi.org/10.1590/So101-47142006000100010>

" Castells, M. 2014. El impacto de internet en la sociedad: una perspectiva global. En C@mbio: 19 ensayos clave acerca de cómo Internet está cambiando nuestras vidas. OpenMind 01-25. <https://www.bbvaopenmind.com/wp-content/ uploads/2014/01/BBVA-OpenMind-libro-Cambio-19-ensayos-fundamentalessobre-c\% $\mathrm{C}_{3} \% \mathrm{~B}_{3}$ mo-internet-est\% $\mathrm{C}_{3} \% \mathrm{~A}_{1}$-cambiando-nuestras-vidas-Tecno$\log \% \mathrm{C}_{3} \% A D a-I n t e r e n t-I n n o v a c i \% C_{3} \% B 3 n . p d f>$ [Consulta: 1 marzo 2020].

»Castilla, Américo, comp. 2010. El museo en escena: política y cultura en América Latina. Buenos Aires: Paidós.

»Fernández Balboa, C. 2009. Casas de cosas: museos, monumentos, y sitios históricos de la Argentina. Buenos Aires: Fundación de Historia Natural de Azara; Ministerio de Educación de la Nación.

" Guía Nacional de Museos. 2013. 3a. ed. Buenos Aires: Secretaría de Cultura. Presidencia de la Nación. <https://issuu.com/secretariadecultura/docs/guianacionaldemuseos $3>$ [Consulta: 12 febrero 2017].

»Laumonier, Isabel. 1993. Museo y sociedad. Buenos Aires: Centro Editor de América Latina.

»Lopes, María Margaret. 2010. Compartir espacios, colgar ballenas y apoyar a las universidades. En Castilla, Américo, comp. El museo en escena: política y cultura en América Latina. Buenos Aires: Paidós. pp 39-52.

»Malosetti Costa, Laura. 2010. Arte e historia. La formación de las colecciones públicas en Buenos Aires. En Castilla, Américo, comp. El museo en escena: política y cultura en América Latina. Buenos Aires: Paidós. pp. 71-88.

»Martín-Barbero, J. 2002. Tecnicidades, identidades y alteridades: desubicaciones y opacidades de la comunicación en el nuevo siglo. En Diálogos de la comunicación. No. 64 .

» Ministerio de Ciencia, Tecnología e Innovación Productiva (MINCYT). 2012. Museo Argentino de Ciencias Naturales 200 años. <http://www.macn.secyt.gov.ar/ descargas/eventos/2012/06/MACN\%20200.pdf> [Consulta: 12 febrero 2017].

" Ministerio de Cultura. Presidencia de la Nación. 2017. Museos. <https://www. cultura.gob.ar/institucional/museos-e-institutos/> [Consulta: 12 febrero 2017].

»Museo Histórico Nacional (MHN). 2020. <http://www.cultura.gob.ar/museos/ museo-historico-nacional/> [Consulta: 15 marzo 2020].

» Museo Nacional de Bellas Artes (MNBA). 2020. <http://www.mnba.gob.ar/museo/historia> [Consulta: 15 marzo 2020].

»Museo de las Escuelas. 2020. Museos pedagógicos y museos escolares. <http:// www.buenosaires.gob.ar/areas/educacion/programas/me/museos.php?menu_ $\mathrm{id}=21429>$ [Consulta 12 febrero 2020]. 
» Podgorny, Irina. 2005. La mirada que pasa: museos, educación pública y visualización de la evidencia científica. En História, Ciências, Saúde - Manguinhos. Vol. 12, suplemento, 231-64.

»Risnicoff de Gorgas, Mónica. 2006. Historia y Museología: los cambios de paradigma. En XXIX Encuentro anual del ICOFOM, Study Series, no. 35, 8 p.

"Ristol, Marcela. 2015. La visibilidad de los museos argentinos en las redes sociales: 2015. Sevilla, UPO, 2015. 206 p. Tesis de Maestría. <http://humadoc.mdp.edu. ar:8o8o/xmlui/handle/123456789/171>

"Todo ciencia.com.ar. 2020. Murió en 1914, Adolfo P. Carranza, creador del Museo Histórico Nacional de la Argentina <http://www.todociencia.com.ar/murio-en1914-adolfo-p-carranza-creador-del-museo-historico-nacional-de-la-argentina/> [Consulta: 1 enero 2018].

»Universidad Nacional de La Plata (UNLP). Facultad de Ciencias Naturales y Museo. 2020. Historia. <http://www.fcnym.unlp.edu.ar/historia> [Consulta 1 marzo 2020].

»Weissel, Marcelo. 2014. La expansión de la Museología en Argentina. En ADIMRA: Buenos Aires. Año XI, no. 11. <http://museomaritimo.com/adimra/Actividades/Revistas/abr_2014.pdf> [Consulta: 12 enero 2020]. 
